\title{
Continuous Positive Airway Pressure or High Flow Nasal Cannula for Respiratory Distress Syndrome: a Randomized Control Trail among Premature Infants
}

\section{Tahereh Esmaeilnia Shirvani}

Tehran University of Medical Sciences

Fatemeh Sadat Nayeri

Tehran University of Medical Sciences

Mamak Shariat

Tehran University of Medical Sciences

Nikoo Nik Nafas

Tehran University of Medical Sciences

Seyyed Reza Mirjalili

Shaheed Beheshti University of Medical Sciences

Seyyed Nasrollah Hosseini ( $\sim$ hoseyniseyyed@yahoo.com )

Tehran University of Medical Sciences

Vafa Ghorban Sabagh

Tehran University of Medical Sciences

Research article

Keywords: Continuous Positive Airway Pressure; High Flow Nasal Cannula; Respiratory Distress Syndrome.

Posted Date: July 25th, 2019

DOI: https://doi.org/10.21203/rs.2.11296/v1

License: (c) (1) This work is licensed under a Creative Commons Attribution 4.0 International License. Read Full License 


\section{Abstract}

Background Respiratory distress syndrome (RDS) is a life-threatening pulmonary disorder which mainly related to the premature infants. This study was conducted to evaluate Continuous Positive Airway Pressure (CPAP) and High Flow Nasal Cannula (HFNC) intervention effectiveness among premature infants with RDS. Methods Overall, 60 preterm infants with RDS at Valiasr Hospital, Tehran, during 2018 in this randomized control trail participated as CPAP and HFNC groups' treatment. CPAP group received the nose with a peep of 4-6 centimeters from birth and continued to improve respiratory distress and the need for oxygen (02). HFNC group received hot and humid flowing 2 to 5 liters/min in nose cannula, until respiratory distress and 02 needing to be resolved. Short clinical outcomes were compared between two groups. Data were analyzed by SPSS version 16 using t-test and logistic regression statistical tests at 95\% significant level. Results There were no significant differences in Pneumothorax, PDA, chronic lung disease, surfactant injection, tracheal intubation, death, NEC, number of full feeding days, duration of hospitalization, and number of oxygen therapy days between the both CPAP and HFNC groups. Conclusions CPAP and HFNC techniques have the same efficacy in confronting RDS in neonates and there is no difference between the two techniques in terms of in-hospital clinical outcomes. However, considering the same efficacy of two methods and less invasive of HFNC compared with CPAP, we concluded this method can be recommended for the improvement of respiratory distress in newborns. Trial Registration Iranian Registry of Clinical Trials identifier IRCT20190623043988N1 Registered 201907-05.

\section{Background}

Respiratory distress syndrome (RDS) is a life-threatening pulmonary disorder which mainly related to the premature infants (1). RDS can be followed by several complications such as chronic lung disease (2) and increasing the mortality among infants (3). As well as, RDS have high economic costs; for example the cost of care for infants with RDS has been reported at $\$ 4.4$ billion a year in the United States (4). Mechanical ventilation (MV) is one of the treatment methods of RDS; however, MV cannot always provide effective oxygenation and stable lung mechanics and maybe followed several side effects such as Pneumothorax, collapsed lung and laryngeal edema $(5,6)$.

Although MV is one of the main methods for treating RDS with surfactant, neonates with mechanical ventilation are always at risk for lung injury, and in some cases, up to $30 \%$ of these patients have chronic pulmonary disease and sometimes lung damage is so severe that it impairs the growth and development of the neonate (7).

Therefore, therapeutic approaches should be sought with minimal clinical complications; as well as, studies have shown that the use of Continuous Positive Airway Compared (CPAP), if the first minutes after birth, accompanied by a reduction in the use of mechanical ventilation, can begin to reduce the chance of death, reduce complications of the disease, such as broncho-pulmonary dysplasia, and also reduce brain hemorrhage (8-10). This method is designed to provide oxygen to the predetermined 
pressure to breathe airborne neonatal airways, and its main purpose is to apply minimum relaxation pressure during the respiratory cycle, to prevent collapse of alveoli and airways (especially when exhaling) preventing (11).

The CPAP was proposed by Gregoryet al in 1971, and is a non-invasive method of respiratory treatment which can be delivered without end tracheal intubation and by given that a continuous level of positive pressure to airways that distends the lungs which that led to promotion of ventilation (12). CAPA causing increasing functional residual capacity which causes air flow inside airbag deployment and with reduced the intrapulmonary shunt causing increased pulmonary capacity (13). This method has several positive effects such as stabilize breathing pattern, reduce respiratory apnea and airway resistance among premature infants (14). It should be noted that unnecessary use of CPAP can be followed several complications such as air leak syndromes, intraventricular hemorrhage (IVH), decreased cardiac output, as well as effects on digestive system and abdominal distension $(15,16)$.

Poisoning is one of the most important complications of early-onset oxygen that occurs quickly, and premature retinopathy is also a serious complication of prolonged use and high oxygen concentrations (11). The use of nasal CPAP is considered as one of the non-invasive methods of respiratory support and its beneficial effects have been proven; nevertheless, the use of this method also has several limitations and clinical problems such as, mechanical problems in placing prong nasal in the nasal holes of premature infants, keep these prongsin a convenient location, trauma to nasal septum, and intolerance to the NCPAP by the infants $(17,18)$.

For this reason, in recent years, researchers have proposed another way to apply PEEP to infants with RDS, which is the use of high-flowing nasal cannula (HFNC), and it widely is increasing (19). The basis of HFNC is to warm and moisturize the gas (preventing the loss of airborne moisture, airway cooling, and thickening of the discharge, that provides high currents without dryness and bleeding in the nose, allowing high currents flow without dry and nosebleeds) and transferring it without a connector extras are only nasal cannula to the infant (20). Also, placing a lighter and easier canola in comparison to the NCPAP may be followed positive result in less damage to the infant's nose and allow the caregiver and the infant's family to work more easily with the infant's (21).

HFNC is a non-invasive respiratory supportive approach that enters the body of warm and humid gas at a temperature close to body temperature with a flow rate of $0.2-0.8 \mathrm{~L} / \mathrm{min}$ (22). Several studies have indicated the efficacy of this method in early treatment of RDS among premature infants (21-23).

However, today's challenge is to choose the best method to achieve positive end-expiratory pressure (PEEP) in the neonate, with the least side-effect and the best clinical outcome for treatment for a newborn with RDS; the main goal is to choose the most non-invasive, most effective and most effective method of respiratory support (24).

Based on the abovementioned background, the objective of this study was comparison of the efficacy of CPAP and HFNC methods in the treatment of Iranian premature infants with RDS. 


\section{Methods}

\section{Participants}

This randomized control trail study was conducted among 60 infants suffering from RDS in Imam Khomeini Hospital Complex, Valiasr Hospital, in Tehran city, during 2018. Infantsin the neonatal intensive care unit (NICU) of Valiasr Hospital participated in the study. Overall, 60 infants were randomly divided in two CPAP and HFNC treatment group.

\section{Inclusion and Exclusion Criteria}

Only the infant's with mild to moderate RDS, birth weight less than 2000 grams, gestational age less than 34 weeks, respiratory severity between 4 to 7 score, and requires oxygen $60 \%$ or less were eligible to participate in this study. As well as, infants who had heart, digestive and respiratory anomalies, intraventricular hemorrhage (IVH) at birth, persistent air leak, sepsis positive at hospitalization in NICU, and Apgar score of less than 5 at 5 minutes were considered to exclusion criteria in this study.

\section{Procedure}

Newborns were enrolled at birth and diagnosis of RDS. Before the arrival of the infants, informed consent was obtained from the parents of the infant's newborn. Before the implementation of the program, the neonates in the two groups were evaluated for the underlying variables and clinical conditions such as: mothers age, weight, length, baby's head circumference, gestational age, apgar at 1 minute after birth, apgar at 5 minutes after birth, and need of oxygen) were considered in this regard mach. Chest X-ray was used to reject other differential diagnosis of respiratory distress. Brain sonography was also used to diagnose ventricular hemorrhage. Both treatments method were performed by one pediatrician for infants.

CPAP treatment was 4 to $6 \mathrm{~cm}$ water and $\mathrm{FIO} 2$ equivalent to $30 \%$ to improve respiratory distress and to reduce the need for oxygen for newborns. The positive pressure created by the device for the infants was measured by a manometer at the beginning of the respiratory tube expiratory arm. It should be noted that treatment was carried out on infants in supine position.

Infants in another group were treated with HFNC methods, and this method continued to improve respiratory distress and eliminate the need for oxygen. Oxygen was heated with a blender unit with a temperature of 32 to $35^{\circ} \mathrm{C}$, and the infant's mouthwaskeptopen during the use of the upper flow. Regulation flow was calculated based on the following formula: flow rate (liters per minute) $=$ weight (kilograms) \& $0.86+0.92$

In both treatment groups, the administration of surfactant was $80 \mathrm{mg} / \mathrm{kg}$ and prior to administration of surfactant, the Arterial Blood Gas (ABG) test was performed. As well as, when the infants have no signs of respiratory and tachycardia, and the need for auxiliary oxygen to $25 \%$ or less, it was considered as the time to stop respiratory support. 


\section{Treatment failure}

Therapeutic failure was defined as increasing oxygen demand up to 96 hours after birth and requiring intubation with $\mathrm{PH}<7.25, \mathrm{Pco} 2>60, \mathrm{PaO} 2<50$ and Fio2 $>60 \%$.

\section{Measures}

Data was collected by trained nurse and included, infant sex (male, female), gestational age (week), mothers age (years), infants weight (gram), infant length (centimeter), baby's head circumference (centimeter), Apgar at 1 minute after birth (score), Apgar at 5 minute after birth (score), Pneumothorax (yes, no), Patent Ductus Arteriosus-PDA (yes, no), chronic lung disease (yes, no), surfactant injection (yes, no), tracheal intubation (yes, no), death (yes, no), necrotizing enterocolitis -NEC (yes, no), number until full feeding days (days), duration of hospitalization (days), number of oxygen therapy days (days) and testament methods (CPAP, HFNC).

\section{Ethical Approval}

Ethical approval was obtained from the research ethics board of the Tehran University of medical sciences (IR.TUMS.IKHC.RCRT.1395.1477).

\section{Data Analyses}

Data analyses were conducted by using SPSS-16. Independent t-test and logistic regression were employed to determine comparability of the CPAP testament group in compare with HFNC group.

\section{Results}

The mean mothers age of infants was 32.16 years [SD: 5.73], ranged from 18 to 47 years. The CAPA group included $15(50 \%)$ girl infants and $15(50 \%)$ boy infants, as well as, the HFNC group included 17 $(56.7 \%)$ female and 13 (43.3\%) male infants., there was no significant differences in sex between two groups $(P=0.605)$. Additionally, Table 1 shows frequency of infants' sex, gestational age, mothers' age, infants' weight, length, baby's head circumference, Apgar at 1 minute after birth, and Apgar at 5 minute after birth in the both CPAP and HFNC groups.

Table 2 showed comparison of full feeding days, duration of hospitalization, and oxygen therapy days between CPAP and HFNC groups. Our findings indicated no significant differences in number of full feeding days, duration of hospitalization, and number of oxygen therapy days between the both CPAP and HFNC groups.

Finally, assessment of relation of clinical conditions with "treatment method" (Table 3) was done by used of logistic regression analyses. As it mentioned in the table 3, there was no significant differences in Pneumothorax, PDA, chronic lung disease, surfactant injection, tracheal intubation, and death, and NEC, between the both CPAP and HFNC groups. 


\section{Discussion}

As previously described, CPAP and HFNC are new methods for RDS support in infants, which each of them has some strengths and limitations. The aim of this study was to comparison of the efficacy of CPAP and HFNC methods in the treatment of Iranian premature infants with RDS. The results of the study indicated there was no difference in clinical conditions between the both CPAP and HFNC groups. This finding is similar to the findings reported by other studies and various studies have shown that both CPAP and HFNC techniques have the same therapeutic effect (25-29). Evidence from other researches supports our findings. However, some studies have shown some differences in the efficacy of these two methods in treatment of RDS among premature infants. For example, Vitaliti et al carried out a research with aim of identify the most efficient treatment of RDS (CPAP or HFNC) in children and indicated both CPAP and HFNC techniques were efficient to improve the clinical conditions, although CPAP was more efficient and rapid compared with HFNC (28). Sreenan et al in their study indicated no significant differences between NCPAP compared with HFNC in apnea and bradycardia among neonates (26). Also, Fernandez-Alvarez et al in their study indicated the clinical conditions of the HHFNC and NCPAP didn't show a significant difference, however,inverse NCPAP, HHFNC does not raise the hazard of nasal trauma (25). Of course, unlike of our findings, the duration of hospitalization among infants in HFNC treatment group was significantly higher than that of infants in CPAP treatment group (29).

However, due to the no significant difference between the two techniques in terms of therapeutic outcomes, the use of any technique can be considered depending on the expert's opinion. Of course, in this regard, the initial conditions of the neonate, the experience and the manipulation of the expert, the access to technical tools and, on the other hand, the cost-effectiveness of the selected technique will be very effective in prioritizing the therapeutic approach. Of course it should be noted our findings indicated that the odds ratio for the clinical outcomes such as tracheal intubation, death, surfactant injection, and NEC in the HFNC method is lower compared to the CPAP method. Moreover, previous studies have also shown that HFNC is less invasive compared with CPAP. Therefore, considering the same efficacy, this method is recommended for the improvement of respiratory distress in newborns.

Our findings have shown that CPAP and HFNC methods in improving clinical outcomes among preterm infants with RDS were have similar findings. Thus, our result indicated based on the expert's opinion, each of the above methods can be used in premature infants with RDS. Of course, in this regard, the infants' initial conditions, medical doctors' experience, access to method tools, and cost-effectiveness of the selected method should be considered in prioritizing the therapeutic approach. However, long-term follow-up of infants treated with these two methods is recommended in a larger sample size.

Results reported in this study have certain limitations. First, low sample size, which was does not provide enough power to study in the final conclusion of the findings or its generalization to the community. Second, lack of consideration of some of the underlying variables, including the level of medical doctors' experienced in performing the CPAP and HFNC methods or disorders during pregnancy among mothers. 


\section{Conclusions}

As a final conclusion, both of CPAP and HFNC techniques have the same efficacy in confronting ARDS in neonates and there is no difference between the two techniques in terms of in-hospital clinical outcomes. However, considering the same efficacy of two methods and less invasive of HFNC compared with CPAP, we concluded this method can be recommended for the improvement of respiratory distress in newborns.

\section{Abbreviations}

RDS: Respiratory distress syndrome

CPAP: Continuous Positive Airway Pressure

HFNC: High Flow Nasal Cannula

02: Oxygen

MV: Mechanical ventilation

NICU: Infantsin the neonatal intensive care unit

IVH: Intraventricular hemorrhage

ABG: Arterial Blood Gas

\section{Declarations}

\section{Ethics approval and consent to participate}

Parents of infants signed the consent form and voluntarily agreed to participate in the study, and this study which has been approved by the Ethics Committee of the Tehran University of Medical Sciences, Tehran, Iran. (IR.TUMS.IKHC.REC.1395.1477).

\section{Consent to publish}

Not applicable.

\section{Availability of data and material}

Please contact the corresponding author for data requests.

\section{Competing interests}

The authors have no conflicts of interest to declare.

\section{Funding}


All aspects of this study, data collection, analysis, and interpretation of data, were supported by Tehran University of Medical Sciences.

\section{Authors' contributions}

TES and SNH contributed to the conception and design of the research; FSN, MS and NNN contributed to the acquisition and analysis of the data; SNH and VGS contributed to the analysis and interpretation of the data; TES, SNH, FSN, XF MS, NNN and VGS contributed to the acquisition, analysis, and interpretation of the data; All authors approved the final manuscript.

\section{Acknowledgements}

The authors would like to thank Imam Khomeini Hospital Complex, Valiasr Hospital, Tehran, Iran.

\section{Authors' Information}

Tahereh Esmaeilnia Shirvani; Associate Professor of Neonatal-Perinatal Medicine.

Fatemeh Sadat Nayeri; Professor of Neonatal-Perinatal Medicine.

Mamak Shariat; Professor of Mother and Child Health.

Nikoo Nik Nafas; Assistant Professor of Neonatal-Perinatal Medicine.

Seyyed Reza Mirjalili; Assistant Professor of Neonatal-Perinatal Medicine.

Seyyed Nasrollah Hosseini; Assistant Professor of Neonatal-Perinatal Medicine.

Vafa Ghorban Sabagh; Neonatal-Perinatal Medicine Expert.

\section{References}

1. Matthay MA, Ware LB, Zimmerman GA. The acute respiratory distress syndrome. The Journal of clinical investigation. 2012; 122(8):2731-40.

2. Lampland AL, Plumm B, Meyers PA, Worwa CT, Mammel MC. Observational study of humidified highflow nasal cannula compared with nasal continuous positive airway pressure. The Journal of pediatrics. 2009; 154(2):177-82.

3. Lipsten E, Aghai ZH, Pyson KH, Saslow JG, Nakhla T, Long J, et al. Work of breathing during nasal continuous positive airway pressure in preterm infants: A comparison of bubble vs variable-flow devices. J Perinatol 2005; 25(7): 453-8.

4. Shoemaker MT, Pierce MR, Yoder BA, DiGeronimo RJ. High flow nasal cannula versus nasal CPAP for neonatal respiratory disease: a retrospective study. Journal of perinatology. 2007; 27(2):85-91. 
5. Wang C, Guo L, Chi C, Wang X, Guo L, Wang W, Zhao N, Wang Y, Zhang Z, Li E. Mechanical ventilation modes for respiratory distress syndrome in infants: a systematic review and network meta-analysis. Critical Care. 2015; 19(1):108.

6. Ware LB, Matthay MA. The acute respiratory distress syndrome. New England Journal of Medicine. 2000; 342(18):1334-49.

7. WANG, Changsong. Mechanical ventilation modes for respiratory distress syndrome in infants: a systematic review and network meta-analysis. Critical Care, 2015, 19.1: 108.

8. Zhu XW, Zhao JN, Tang SF, Yan J, Shi Y. Noninvasive high-frequency oscillatory ventilation versus nasal continuous positive airway pressure in preterm infants with moderate-severe respiratory distress syndrome: a preliminary report. Pediatric pulmonology. 2017; 52(8):1038-42.

9. Shin J, Park K, Lee EH, Choi BM. Humidified high flow nasal cannula versus nasal continuous positive airway pressure as an initial respiratory support in preterm infants with respiratory distress: a randomized, controlled non-inferiority trial. Journal of Korean medical science. 2017; 32(4):650-5.

10. XU, Huajun, Yi Hongliang, Guan Jian, Yin Shankai. Effect of continuous positive airway pressure on lipid profile in patients with obstructive sleep apnea syndrome: a meta-analysis of randomized controlled trials. Atherosclerosis, 2014, 234.2: 446-453.

11. Sasi A, Malhotra A. High flow nasal cannula for continuous positive airway pressure weaning in preterm neonates: A single-centre experience. Journal of paediatrics and child health, 2015, 51.2: 199-203.

12. Dewez JE, van den Broek N. Continuous positive airway pressure (CPAP) to treat respiratory distress in newborns in low-and middle-income countries. Tropical doctor. 2017; 47(1):19-22.

13. Elgellab A, Riou Y, Abbazine A, Truffert P, Matran R, Lequien P, Storme L. Effects of nasal continuous positive airway pressure (NCPAP) on breathing pattern in spontaneously breathing premature newborn infants. Intensive care medicine. 2001; 27(11):1782-7.

14. Miksch RM, Armbrust S, Pahnke J, Fusch C. Outcome of very low birthweight infants after introducing a new standard regime with the early use of nasal CPAP. Eur J Pediatr 2008; 167(8): 90916

15. Ali H, Esampalli S, Aldridge L, Lal M. 1231 Pneumothorax and Air Leak Syndromes in the Newborn, a Cohort Study. Pediatric Research. 2010; 68(S1):610.

16. Jatana KR, Oplatek A, Stein M, Phillips G, Kang DR, Elmaraghy CA. Effects of nasal continuous positive airway pressure and cannula use in the neonatal intensive care unit setting. Archives of Otolaryngology-Head \& Neck Surgery. 2010; 136(3):287-91.

17. McCoskey L. Nursing care guidelines for prevention of nasal breakdown in neonates receiving nasal CPAP. Advances in Neonatal Care. 2008; 8(2):116-24.

18. Nascimento RM, Ferreira AL, Coutinho AC, Veríssimo RC. The frequency of nasal injury in newborns due to the use of continuous positive airway pressure with prongs. Revista latino-americana de enfermagem. 2009; 17(4):489-94. 
19. Frat JP, Thille AW, Mercat A, Girault C, Ragot S, Perbet S, Prat G, Boulain T, Morawiec E, Cottereau A, Devaquet J. High-flow oxygen through nasal cannula in acute hypoxemic respiratory failure. New England Journal of Medicine. 2015; 372(23):2185-96.

20. Saslow JG, Aghai ZH, Nakhla TA, Hart JJ, Lawrysh R, Stahl GE, et al. Work of breathing using highflow nasal cannula in preterm infants. J Perinatol 2006; 26(8): 476-80

21. Fernandez-Alvarez JR, Gandhi RS, Amess P, Mahoney L, Watkins R, Rabe H. Heated humidified highflow nasal cannula versus low-flow nasal cannula as weaning mode from nasal CPAP in infants $\leq 28$ weeks of gestation. European journal of pediatrics. 2014; 173(1):93-8.

22. Sreenan C, Lemke RP, Hudson-Mason A, Osiovich H. High-flow nasal cannulae in the management of apnea of prematurity: a comparison with conventional nasal continuous positive airway pressure. Pediatrics. 2001;107(5):1081-3.

23. Holleman-Duray D, Kaupie D, Weiss MG. Heated humidified high-flow nasal cannula: use and a neonatal early extubation protocol. Journal of perinatology. $2007 ; 27(12): 776-81$.

24. Jobe AH, Kramer BW, Moss TJ, Newnham JP, Ikegami M. Decreased indicators of lung injury with continuous positive expiratory pressure in preterm lambs. Pediatric research. 2002; 52(3):387.

25. Fernandez-Alvarez JR, Gandhi RS, Amess P, Mahoney L, Watkins R, Rabe H. Heated humidified highflow nasal cannula versus low-flow nasal cannula as weaning mode from nasal CPAP in infants $\leq 28$ weeks of gestation. European journal of pediatrics. 2014; 173(1):93-8.

26. Sreenan C, Lemke RP, Hudson-Mason A, Osiovich H. High-flow nasal cannulae in the management of apnea of prematurity: a comparison with conventional nasal continuous positive airway pressure. Pediatrics. 2001;107(5):1081-3.

27. Holleman-Duray D, Kaupie D, Weiss MG. Heated humidified high-flow nasal cannula: use and a neonatal early extubation protocol. Journal of perinatology. 2007; 27(12):776-81.

28. Vitaliti G, Vitaliti MC, Finocchiaro MC, Di Stefano VA, Pavone P, Matin N, Motamed-Gorji N, Lubrano R, Falsaperla R. Randomized comparison of helmet CPAP versus high-flow nasal cannula oxygen in pediatric respiratory distress. Respiratory care. 2017; 62(8):1036-1042.

29. Yoder BA, Stoddard RA, Li M, King J, Dirnberger DR, Abbasi S. Heated, humidified high-flow nasal cannula versus nasal CPAP for respiratory support in neonates. Pediatrics. 131(5):e1482-90

\section{Tables}

Table 1: Pretest Equivalency results for CPAP $(n=30)$ and HFNC groups $(n=30)$ 


\begin{tabular}{llll}
\hline Variables & CPAP Group & HFNC Group & $P$-value \\
& Mean $( \pm$ SD) & Mean $( \pm$ SD) & \\
\hline Mothers Age (years) & $31.57(4.72)$ & $32.79(6.69)$ & 0.424 \\
Weight (g) & $1315.67(417.49)$ & $1181.17(306.31)$ & 0.160 \\
Length (cm) & $39.17(4.99)$ & $39.00(4.89)$ & 0.897 \\
Baby's head circumference (cm) & $27.71(2.73)$ & $26.82(2.63)$ & 0.199 \\
Gestational age (week) & $29.50(2.09)$ & $30.40(2.08)$ & 0.065 \\
Apgar at 1 minute after birth & $6.80(1.99)$ & $6.14(2.91)$ & 0.863 \\
Apgar at 5 minute after birth & $8.40(1.22)$ & $8.17(1.34)$ & 0.638 \\
\hline
\end{tabular}

Table 2: Comparison of full feeding days, duration of hospitalization, and oxygen therapy days between CPAP and HFNC groups

\begin{tabular}{llll}
\hline Variables & CPAP Group & HFNC Group & $P$-value \\
& Mean $( \pm$ SD) & Mean $( \pm$ SD) & \\
\hline Full feeding days & $18.50(10.57)$ & $18.73(8.05)$ & 0.930 \\
Duration of hospitalization & $32.53(19.86)$ & $41.27(22.46)$ & 0.116 \\
Oxygen therapy days & $10.34(15.62)$ & $14.76(22.81)$ & 0.839 \\
\hline
\end{tabular}

Table 3: Multiple logistic regression analysis for clinical conditions related to CPAP and HFNC 


\begin{tabular}{|c|c|c|}
\hline Clinical Conditions & Odds Ratio (95\% CI) & $P$ value \\
\hline \multicolumn{3}{|l|}{ Pneumothorax } \\
\hline CPAP & 1.00 & 0.561 \\
\hline HFNC & $2.071(0.178-24.148)$ & \\
\hline \multicolumn{3}{|l|}{$P D A$} \\
\hline CPAP & 1.00 & 0.775 \\
\hline HFNC & $1.179(0.383-3.629)$ & \\
\hline \multicolumn{3}{|c|}{ Chronic lung disease } \\
\hline CPAP & 1.00 & 1.000 \\
\hline HFNC & 1. $00(0.302-3.308)$ & \\
\hline \multicolumn{3}{|l|}{ Surfactant injection } \\
\hline CPAP & 1.00 & 0.837 \\
\hline HFNC & $0.837(0.260-2.699)$ & \\
\hline \multicolumn{3}{|l|}{ Tracheal intubation } \\
\hline CPAP & 1.00 & 0.505 \\
\hline HFNC & $0.322(0.131-1.951)$ & \\
\hline \multicolumn{3}{|l|}{ Death } \\
\hline CPAP & 1.00 & 0.728 \\
\hline HFNC & $0.781(0.195-3.137)$ & \\
\hline \multicolumn{3}{|l|}{$N E C$} \\
\hline CPAP & 1.00 & 0.452 \\
\hline HFNC & $0.556(0.120-2.569)$ & \\
\hline
\end{tabular}

